\title{
e-Migrinter
}

6 | 2010

Rroms \& Gens du Voyage

\section{Rejets éternels : les collectivités locales face aux Tsiganes et aux nomades}

Jean-Pierre Liégeois

\section{OpenEdition}

Journals

Édition électronique

URL : https://journals.openedition.org/e-migrinter/1474

DOI : 10.4000/e-migrinter. 1474

ISSN : 1961-9685

Éditeur

UMR 7301 - Migrinter

Édition imprimée

Date de publication : 15 octobre 2010

Pagination : 5-28

ISSN : 1961-9685

\section{Référence électronique}

Jean-Pierre Liégeois, "Rejets éternels : les collectivités locales face aux Tsiganes et aux nomades 》, eMigrinter [En ligne], 6| 2010, mis en ligne le 06 novembre 2019, consulté le 20 mai 2021. URL : http:// journals.openedition.org/e-migrinter/1474 ; DOI : https://doi.org/10.4000/e-migrinter.1474 
DOSSIER

\title{
Rejets éternels : les collectivités locales face aux Tsiganes et aux nomades
}

\author{
Jean-Pierre Liégeois
}

\begin{abstract}
Note introductive
Ce texte a été publié en 1981 dans le numéro 28 de la Revue Pluriel-débat (Cedrasemi-CNRS). Son titre était prémonitoire, ou tout simplement pertinent, car les observations faites et les analyses présentées peuvent être (re)lues trente ans plus tard comme toujours actuelles. Pour ce numéro spécial de e-migrinter concernant notamment les dispositifs d'accueil proposés aux nomades, rien n'a été changé et le texte est (re)publié à l'identique, y compris pour le titre et les notes.

Ce texte a été envoyé à la Revue avant les déclarations du chef de l'Etat et les dispositions prises en juillet 2010 concernant les Roms et les "Gens du voyage », qui illustrent plus que nécessaire les tendances analysées...
\end{abstract}

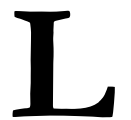

es pages qui suivent présentent une typologie des attitudes des collectivités locales face aux Tsiganes et nomades, et une analyse de la fonction que remplit actuellement pour ces collectivités l'ouverture « d'aires de stationnement » pour les nomades. On verra que, dans l'ensemble, si la politique des pouvoirs publics se veut assimilatrice depuis peu (comme nous l'avons montré dans Pluriel, $\mathrm{n}^{\circ} 19,1979$, et dans Esprit de mai 1980), celle des autorités locales en reste à cette autre forme de négation qu'est le rejet, ou la réclusion dans des terrains contrôlés.
Les attitudes et comportements analysés touchent quotidiennement entre 100000 et plus de 200000 personnes en France, selon que l'on considère ceux qui vivent toute l'année en nomades, ou que l'on y ajoute ceux qui - Tsiganes sédentarisés par exemple - voyagent par intermittence, et encore ceux qui, échoués dans un bidonville ou propriétaires d'un terrain sur lequel ils souhaitent séjourner, sont toujours rejetés. Le nombre des exemples donnés n'est malheureusement pas exhaustif, mais simplement illustratif des procédés suivis face à une minorité durement maltraitée, en France comme ailleurs.

Le rejet séculaire

Quelquefois les ordonnances et édits des pouvoirs centraux laissent apparaître, comme prétexte à la rigueur de la loi, certaines plaintes et doléances de la part des populations. Et, pour le bienfait des populations, il convient d'écarter les Bohémiens, ce désordre étant commun dans la plupart des provinces de notre Royaume, et d'autant qu'il importe au repos de nos sujets et de la tranquillité publique de renouveler les anciennes 
ordonnances à l'égard des dits Bohêmes ... ${ }^{1}$. Ainsi sont rejetés des individus qui risquent de troubler l'ordre en troublant la tranquillité. Mais si l'ordre est important pour les pouvoirs publics, la tranquillité l'est tout autant pour les populations et les autorités locales, qui n'attendent pas la loi pour expulser le nomade.

Paris, aux portes de laquelle se présentent en 1427 une centaine de Tsiganes, les garde moins d'un mois avant de les repousser vers Pontoise. L'année précédente, à Gap, une somme est allouée par la commune à trois habitants de garde aux portes au temps où les Sarrasins étaient dans ladite citée de Gap ${ }^{2}$. Plus tard, il arrive que les habitants soient rassemblés au son du tocsin pour défendre leur ville ou poursuivre les Bohémiens, avec des indemnités de capture ; mais dès le $15 \mathrm{e}$ siècle, même lorsque les groupes tsiganes possèdent des passeports royaux, blesser ou tuer un Bohémien est une faute pardonnable. Les lettres de rémission accordées en décembre 1453 au dénommé Meugin Gineval, de la Cheppe en Champagne, en sont une preuve, et décrivent fort bien le type de contact qui s'établit entre sédentaires et nomades, ces derniers précédés d'une mauvaise réputation.

Au cours des siècles suivants, et de façon de plus en plus marquée, les exemples de rejet se multiplient. Ainsi à une époque où être Bohémien est passible des galères, les notables de Sisteron décident d'expulser les Baumianz qui demandent en 1616, suivant la contume, de loger au lieu de la Baulme pour trois jours. Quelques années plus tôt, en 1611, Angers enjoint aux susdits Egyptiens de déloger et s'esloigner de ceste ville et fauxbourgs de cinq lieux pour le moins dedans ce jour à peine de punition corporelle. L'ordre avait déjà été donné

\footnotetext{
${ }^{1}$ Louis XIV, Déclaration du Roy rendue contre les Bohêmes et ceux qui leur donnent retraite, 11 juillet 1682.

2 Cf. François de Vaux de Foletier, Les Tsiganes dans l'Ancienne France, Connaissance du Monde, 1961, p. 19. «Sarrasins" est un nom souvent donné aux Tsiganes à cette époque.
}

en 1499 et $15488^{3}$. Toulon en 1619 refuse les Bohémiens, les Égyptiens, les vagabonds et gens sans aveu, par des interdictions préventives. En 1672 Vannes fait très expresses et itératives défens à toutes personnes se disants Égyptiens ou bohémiens de loger en cette ville et faux bourgs ${ }^{4}$.

Rares sont les agglomérations qui, comme en Espagne après la pragmatique de 1746, demandent que les Gitans demeurent, car s'ils partent c'est au grand préjudice des agriculteurs qui se trouvent sans forgerons pour entretenir leurs outils de travail. Il est reconnu par exemple qu'en se débarrassant de Francisco de Vargas, de Villafranca, homme calme et pacifique [...] employé pour son travail de forgeron [...] on avait porté un très notable préjudice aux habitants de ladite ville, et spécialement aux agriculteurs dont certains avaient perdu de nombreuses heures de travail. En conséquence il est demandé que Francisco de Vargas puisse revenir exercer son métier. La même requête a lieu à Velez et à Motril où le procureur général déclare lui aussi les Gitans d'utilité publique. Des villages espagnols demandent que soient délivrés aux Gitans des passeports pour qu'ils puissent venir animer les fêtes ${ }^{5}$. Mais le contexte espagnol de longue sédentarisation diffère à cette époque de la politique de rejet pratiquée en France jusque dans la première moitié du 20e siècle. Et si, dans l'un comme dans l'autre des pays, au début les groupes tsiganes sont bien reçus, c'est parce qu'ils sont porteurs de lettres de recommandation de tel ou tel roi, ou d'une bulle du pape les certifiant pèlerins. C'est pourquoi lors de leur premier passage dans la ville de Murcie, en Espagne, les membres du Conseil de la ville firent don en 1470 au comte Jacques de Petite Égypte de deux mille maravedis pour lui fournir aide et aumône pour les frais de voyage.

\footnotetext{
3 Cité par Henriette Asséo, «Le traitement administratif des Bohémiens", in Problèmes socioculturels en France au XVIIe siècle, Ed. Klineksieck, 1974, p. 57.

${ }^{4}$ Ibid.

5 Archives municipales de Murcie, legajo n ${ }^{\circ} 4150$. Sur l'Espagne, cf . J.-P. Liégeois, "Gitans et pouvoirs publics en Espagne », Ethno-psychologie , n 1, 1980.
} 
Le comte était de retour de Saint-Jacques de Compostelle et s'en retournait vers son pays dont il est précisé qu'il était fort loin. En 1471, le duc Paul de Petite-Égypte se voit offrir mille maravedis du même Conseil de Murcie. On doit noter que, le Conseil n'ayant pas les fonds nécessaires à ce don, c'est le maire Magaz Ferrandez qui en fait l'avance au trésorier ${ }^{6}$. De la même façon, à Tournai, en 1430, les autorités locales offrent de l'argent, du blé, des harengs, du vin, de la bière et du bois aux Égyptiens, et font apposer une affiche pour demander à la population de les bien recevoir, les injures à leur égard seront punies ${ }^{7}$. La même année Amiens accorde huit livres tournois à un groupe de passage ${ }^{8}$. Sisteron qui rejettera les Bohémiens à partir de 1499, en octobre 1419 leur permet, sinon d'entrer dans la ville, du moins de s'arrêter à proximité et le trésorier leur apporte cent pains, de la viande de mouton, du vin et de l'avoine pour les chevaux'.

Puis, le temps de l'aumône et de la croyance aux histoires de pèlerinages étant rapidement passé, reste une façon de procéder envers les groupes de Bohémiens qui permet de s'en débarrasser sans violence: c'est un don d'argent ou de victuailles pour déloger ou droit de passade; ce sont quelquefois des tractations réalisées devant notaire. Contre un don, le capitaine des Égyptiens s'engage à passer son chemin avec sa troupe, à faire en sorte qu'aucun méfait ne soit commis et à ne pas revenir avant le délai d'une année en général. Un modus vivendi s'établit. Pour quelques livres de plus ou pour une poignée de deniers, les autorités locales achètent un peu de tranquillité : à travers l'aumône ainsi pervertie, c'est le rejet qui transparait, et de

\footnotetext{
${ }^{6}$ Archives municipales de Murcie, Actes capitulaires. Le fait est signalé par Juan Torres Fontes, Don Pedro Fajardo Adelantado Mayor del Reino de Murcia, Biblioteca Reyes Católicos, Consejo superior de Investigaciones cientificas, Madrid, p. 123, et Compilación de los milagros de Santiago de Diego Rodriguez de Almela, Murcia, 1946, pp. XXXIIXXXIII.

${ }^{7}$ Cité par François de Vaux de Foletier, op. cit., p. 30.

${ }^{8}$ Cité par Henriette Asséo, op. cit., p. 69.

${ }^{9}$ Cité par François de Vaux de Foletier, op. cit., p . 18.
}

leur côté les Bohémiens utilisent la peur qu'ils suscitent à tort ou à raison. Le temps de la surprise lui aussi est passé, comme celui du merveilleux de ces groupes bigarrés et extraordinaires de campeurs chevelus qui apparaissent au début du 15e siècle. Le temps de l'ordre et des marchés commence. Devant notaire en 1603 Jehan de La Grave, cappitaine d'une compagnie de Egiptiens confesse a voir receu de la communauté de Lachau ung escu par les mains de Jehan de Michel, ung des consuls de ce lieu, qu'ils luy ont donné pour ne venir en ce lieu et $q u^{\prime} i l s$ aillent en autre part ${ }^{10}$. Ou en septembre 1636 à Bras, près de Saint Maximin, 20s aux Bouemiens. Le vingt dud. baillés pour fere passer les Indiens de se lieu ${ }^{11}$.

Les populations ne sont plus curieuses devant le bizarre, mais anxieuses face au marginal.

\section{Le rejet simple}

Pour fere passer les Indiens, les autorités locales du $20^{\mathrm{e}}$ siècle procèdent de diverses façons. Il y a d'abord le simple refus des Tsiganes et nomades: les panneaux Stationnement interdit aux nomades qui demeurent malgré leur illégalité. C'est à Saint-Brieuc la juxtaposition des panneaux Place de la Liberté et Le Stationnement est interdit aux nomades et S.D.F. C'est à Gonesse l'apposition d'affiches NON à l'installation d'un camp de GITANS à Gonesse signées Le Comité de Défense de Gonesse.

$\mathrm{Au}$ Pouliguen, au cours d'une réunion du Conseil municipal, il est décidé d'aménager un terrain pouvant accueillir 750 campeurs, à l'usage exclusif des touristes, et il est souligné qu'il n'y a pas de place pour les nomades, au point que le conseil demande qu'une dérogation soit accordée à l'obligation faite aux communes d'assurer le séjour même de vingtquatre heures aux forains et nomades de passage ${ }^{12}$. Le maire et conseiller général d'Argenteuil, ayant entendu parler de l'aménagement d'un

\footnotetext{
${ }^{10}$ Cité par François de Vaux de Foletier, op. cit., p. 67.

${ }^{11}$ Ibid., p. 68.

12 L'Eclair de Nantes, 11 mars 1968.
} 
terrain de stationnement pour les nomades, évoque la nécessité d'assainir sa ville et dénonce la menace que des éléments hétérogènes font peser sur l'agglomération ${ }^{13}$. Si au $16^{\mathrm{e}}$ siècle les populations craignaient que les Tsiganes soient porteurs de la peste, au 20e siècle on les suppose porteurs de troubles, et c'est le prétexte pour les refouler.

Troubles possibles aussi dans l'ordonnancement des sites : au moment où le gouvernement prône la protection des sites, de la nature ou de l'environnement, je pense qu'il fallait avoir le courage de se manifester dans le sens d'une politique plus sévère à l'égard de ces nomades que nous trouvons, chez nous, trop sédentaires, commente le maire de Villelaure, dans le Vaucluse, en prenant un arrêté qui limite à trois jours le stationnement des nomades sur le territoire de sa commune, alors que plusieurs familles ont l' habitude d'y stationner longuement depuis des dizaines d'années. Et les maires utilisent l'arme administrative de rejet qui leur est fournie par la réglementation de l'État relative au stationnement des caravanes.

\section{Le rejet violent}

Au-delà du simple refus des Tsiganes et nomades, il y a de la part des communes un passage à l'acte violent d'expulsion, qui peut aller jusqu'à l'usage d'armes. La légalisation en existait quelquefois auparavant: en 1646, à Berne, une ordonnance est prise contre les Tsiganes et autres Heiden, qui accorde à chacun le droit d'abattre et de liquider lui-même par bastonnade et par coup de fen ceux qui seraient considérés comme suspects ${ }^{14}$. Sans oublier les primes de capture de certaines époques. C'est peutêtre pourquoi, de temps à autre, un sédentaire seul ou non part à la chasse au nomade. A Saint-Gervais-du-Perron, dans l'Orne, le préfet ayant refusé les délibérations et l'arrêté du Conseil municipal

\footnotetext{
${ }^{13}$ La Renaissance d'Argentevil, 9 septembre 1967.

14 Cité par Karl Rinderknecht, «Les Tsiganes en Suisse ", in Tsiganes, nomades mystérieux, Ed. Mondo, 1973 , p. 128.
}

pour interdire le stationnement, un conseiller municipal est allé tirer des coups de fusil en direction des nomades installés près de la décharge publique, pour les faire partir.

Je voudrais vous dire de la façon dont on est mené sur le voyage. Voici : il y a buit ans, ma mère était très malade; ça se passait à $B$., en Dordogne, lorsqu'un docteur lui a recommandé qu'il fallait lui arracher plusieurs dents. Comme elle avait une maladie assez, grave, nous nous sommes arrêtés au Champ de Foire, mais hélas pas pour longtemps. Dix minutes après, un fourgon de police. Ils sont intervenus pour nous faire partir. Il a fallu qu'on se place à sept kilomètres de la ville. Alors, on est revenu avec une seule voiture devant le dentiste et, comme elle avait fait un infarctus du myocarde, nous l'avons accompagnée, moi et mes frères et deux de mes cousins, pour le cas où elle se trowverait mal. Une mère, je crois que c'est bien tout. Alors de nouveau la police se trouve à passer dans cette rue. Ils ont couru vers nous pour nous demander nos papiers, avec des cris si féroces que les personnes qui passaient se sont arrêtées, croyant qu'on était peut-être des bandits. J'ai eu très honte; je leur ai dit qu'on allait donner nos papiers, mais qu'ils pouvaient être polis. De la façon qu'ils ont fait alors, ils nous ont brutalisés pour nous amener au commissariat et, en entrant, le chef a demandé ce qui se passait. Ils nous ont dit: 'Ici maintenant vous êtes chez nous'. Alors ils se sont mis à trois ou quatre pour nous taper et ils nous ont fait faire une heure de cellule, à la place de s'occuper de notre malade. Voilà comment on est traité à la vue du monde. ${ }^{15}$

Aujourd'bui, pour faire cent kilomètres, nous sommes contrôlés trois fois: pièces d'identité, fichier, ça nous tient trois heures jusqu'à ce qu'ils aient terminé. On nous fouille même les caravanes et les camions. On nous ouvre les portes pour voir si on transporte de l'alcool [...]. Un jour le docteur nous avait interdit de partir avec les gosses malades. Malgré l'accord

15 Témoignage tsigane reproduit dans Etudes tsiganes, $n^{\circ} 1,1966$, p. 9 . 
du propriétaire du terrain, les gardes nous ont chassés. On nous prend pour des Indiens, comme en Amérique en 1880 et quelques... On est chassés par les municipalités et les gendarmes viennent à cinq-six avec des fourgons. Une demi-journée à Belfort et on vient déjà avec des mitraillettes. ${ }^{16}$

Des groupes importants en stationnement sont expulsés, par exemple à Noisy-le-Grand en 1965 (environ deux cents familles chassées en novembre par les policiers aidés de chiens) et l'automne suivant à Rosny-sous-Bois, quand plusieurs milliers de personnes trouvent au retour de leur travail leur habitat, précaire mais entretenu, rasé par des engins de chantier, avec tout ce qui se trouve à l'intérieur, et les femmes et les enfants assis au milieu des décombres. Une société immobilière envisageait de construire sur le terrain libéré, mais aucun délai n'avait été notifié aux occupants. Dans une lettre adressée au Mouvement contre le Racisme, l'Antisémitisme et pour la Paix, le préfet demeure laconique: J'ai l'bonneur de vous faire savoir que les mesures prises jusqu'à présent concernant ce campement n'avaient pas pour but d'expulser les occupants mais d'en limiter $l^{\prime}$ extension ${ }^{17}$. Lors de ces actions, la cellule familiale est détruite, apparaissent l'alcoolisme, l'internement psychiatrique, le placement sanitaire des enfants, dûs aux mauvaises conditions de logement ${ }^{18}$.

16 Témoignage d'un Tsigane qui voyage dans les Vosges, reproduit par Monde gitan, n² 26, 1973, p. 5 .

${ }^{17}$ De nombreux détails sont donnés dans la presse de l'époque, en raison de l'ampleur de cette expulsion. Voir aussi La Voix Mondiale Trigane, n 28, avril 1967, pp. 1-8. Les exemples étrangers sont de même nature et les attitudes identiques, quel que soit le pays considéré; la fin reste la même bien que les moyens varient selon les nations et les époques.

18 On a l'exemple d'Avignon, avec le passage du quartier de La Balance vers la Cité du Soleil et Clarefond. Des détails sont donnés par Marie Cannizzo, D'où viens-tu Gitan?, mémoire pour le CAEI d'instituteur, 1980 ; ou encore l'exemple de Grenade, lorsque dans les années soixante, des milliers de Tsiganes du Sacro Monte furent «relogés» dans des baraquements de tôle ou de fibro-ciment, sous le soleil andalou : cf. J.P. Liégeois, "Veillée gitane», Etudes Tsiganes , $\mathrm{n}^{\circ} 3$, 1967 , pp. $10-24$.
Le rejet indirect

Avec le simple rejet et la violence des expulsions, il y a une troisième façon de procéder, de la part des autorités locales, pour se débarrasser des nomades et Tsiganes: c'est le rejet indirect, qui à l'acte de violence substitue des conditions de séjour impossibles. En une époque où, comme cela ressort de l'étude des textes réglementaires, l'humanisme se développe, c'est une manière de faire qui offre plusieurs avantages à celui qui l'utilise.

C'est la juxtaposition classique et symbolique de deux panneaux avec une flèche indiquant la même direction, dont l'un comporte l'inscription «TERRAIN réservé aux NOMADES », et l'autre «DÉPOTOIR MUNICIPAL ».

C'est le détour par l'hygiène et la vaccination, qui en 1979 fait prendre au maire de Charmes, dans les Vosges, l'arrêté suivant: Chaque fois que les personnes visées à l'article 1er (sans domicile ni résidence fixe) se présenteront dans la commune pour y camper ou $y$ stationner, elles devront justifier par un certificat dûment établi qu'elles ont subi les vaccinations et revaccinations obligatoires, à défaut de quoi elles devront se soumettre $\grave{a}$ une vaccination ou revaccination immédiate, ou bien quitter le territoire de la commune. Or les touristes et autres personnes de passage ne sont pas soumis à ce contrôle. Pas plus qu'à Chartèves, dans l'Aisne, où existe la même disposition, et dans bien d'autres communes.

C'est le terrain d'Argentan, entouré d'un mur plein de deux mètres vingt de haut avec une seule ouverture exigüe, qui en fait un ghetto, ou le terrain de Villedomer (Indre-et-Loire) limité à deux cent cinquante mètres carrés et à deux voitures pour quarante-huit heures. A Monthiers et Bézule-Guéry (Aisne), Le stationnement des nomades est autorisé, mais la commune ne possède pas de terrain. Dans le même département, à Brécy le stationnement des nomades est interdit sur tout le territoire de la commune. Pendant les travaux de 
récolte des pois et haricots, il sera permis aux nomades de stationner sur le chemin dit des Vingt Arpents ou de la Justice et à Coincy le stationnement et le campement des nomades ne sont autorisés qu'au lieu-dit Le Fond de Sorel. La durée du campement et du stationnement sur l'emplacement ci-dessus désigné ne pourra dépasser deux mois (août et septembre) pour la durée des travaux saisonniers. Economie locale oblige, comme aux SaintesMaries-de-la-Mer d'où les Tsiganes sont expulsés au lendemain du pèlerinage. Dans l'Aisne encore, Chierry interdit le stationnement aux vagabonds, nomades, roulottiers et romanichels et le tolère pour les forains justifiant de leur profession quoiqu' 'il est expressément défendu aux personnes ci-dessus indiquées de se faire accompagner par des enfants de moins de seize ans. Quant à Nogent l'Arthaud, le stationnement s'y réduit à une halte de douze heures de jour ou d'une seule nuit si la demande en a été faite aux heures d'ouverture de la mairie, qui est fermée aussi le samedi aprèsmidi et le dimanche ${ }^{19}$. A Pouzac (HautePyrénées) le stationnement n'est autorisé que de nuit au bord d'une route. Par un arrêté du 23 mai 1971, le maire de Fauverney (Côted'Or) a limité le stationnement à vingt quatre heures, moyennant une redevance de trente francs à verser à l'arrivée. Le séjour peut se prolonger, mais la redevance doit être versée à nouveau. A Bayonne, par arrêté du 13 septembre 1972, seul le lieu-dit Chemin de l'Estanquet, qui peut contenir au maximum deux caravanes, est autorisé pour le séjour des nomades.

Tous ces actes permettent de conserver une façade d'humanisme. On n'est plus au temps où, comme dans l'Allemagne $\mathrm{du} 18 \mathrm{e}$ siècle ou aux Pays-Bas, des épouvantails à Tsiganes qui figuraient un Tsigane fustigé ou pendu se trouvaient à l'entrée d'une agglomération. Le panneau laqué a remplacé la pancarte de bois, l'écriture la peinture naïve, et l'amende dissuasive le châtiment corporel.

19 Les exemples pour le département de l'Aisne figurent dans Monde gitan, $\mathrm{n}^{\circ}$ 35, 1975.
Le rejet indirect permet de garder bonne conscience, et rend l'action compatible avec l'option d'assimilation des pouvoirs publics. La nuance feutrée du discours administratif laisse place, au niveau des autorités locales, à l'exploitation grossière du jeu de mots qui permet d'interpréter pour abuser. L'art de la nuance devient manigance. Il est facile à celle-ci d'utiliser celui-là : la subtilité des textes réglementaires ouvre la voie à l'ambiguité permissive.

\section{Les affaires de rejet}

Ce n'est pas sa violence ou sa dureté qui rend le rejet ou l'abus excessif ou considéré comme tel, mais sa visibilité. Quand les moyens de communication de masse et par eux l'opinion régionale ou nationale deviennent sensibles aux agissements des autorités ou des populations locales, le rejet quasi routinier se transforme en «affaire ». C'est ainsi qu'à Bron dans le Rhône des comités de défense refusent l'implantation d'un terrain de stationnement pour les nomades et dénoncent la position des élus ${ }^{20}$. A la même époque les habitants du quartier de Longpré (Amiens) publient un communiqué dans Le Courrier picard, pour que les nomades s'éloignent: Quand donc les autorités responsables comprendront-elles que les honnêtes gens, lassés d'assister à l'impunité ou au laisser-aller qui semblent caractériser notre époque, pourraient passer à l'action directe ou collective? A force de songer à se défendre, ils seraient aussi capables de prendre des risques et, à leur tour déclencher des représailles faisant leur cet adage qui dit que la meilleure défense est encore l'attaque. A Toulouse, lorsqu'il s'est agi en 1973 de loger dans de nouveaux bâtiments quelques familles du bidonville du Ginestous une «association de sauvegarde des intérêts de Saint-Martin-de-Touch » a regroupé les futurs voisins et l'affaire, qui traîna presque deux ans, s'est terminée devant le Conseil d'État. Dans d'autres pays, les exemples sont identiques.

\footnotetext{
${ }^{20}$ Le Journal Quotidien Rhône-Alpes, 11 janvier 1979.
} 
En 1971, à Dung, dans le Doubs, une famille tsigane qui demande à être logée en H.L.M. est repoussée et on déclare qu' il est impossible de loger des Gitans au milien de la population. Le maire explique que ce ne sont peut-être pas de mauvaises gens, mais personne n'en veut ici. A Helfranzkirch (Haut-Rhin), la commune refuse en 1968 l'installation d'une famille de dix enfants. Le maire écrit au sous-préfet de Mulhouse : Le conseil municipal $d^{\prime}$ Helfranzkirch se demande quelle responsabilité $M$. le sous-préfet veut imposer à la commune. Implanter cette famille de Gitans dans la commune, soit en cédant une parcelle de terrain, soit en lui offrant un logement équivaudrait à attirer d'autres nomades encore. En effet, depuis le début de l'automne, deux autres familles de Gitans, parents de la famille Reinhardt, se sont incrustés dans le ban. Le maire déclare au journal local que si la souspréfecture l'oblige à conserver cette famille, le conseil municipal démissionnera collectivement $^{21}$ et après intervention du préfet, il répond en mars que la municipalité maintiendra sa position, estimant que l'on ne peut imposer à la commune une famille appartenant à un groupe social itinérant. Le maire avait quelques années avant, en faisant atteler un tracteur à une roulotte, conduit la famille Reinhardt hors de la commune sur un terrain froid et malsain, à un kilomètre cinq cents d'un point d'eau et à quatre kilomètres de l'école ${ }^{22}$.

A Schalkendorf (Bas-Rhin), la famille tsigane Weiss avait acheté une ancienne ferme. Mais dès l'installation de la famille, l'hostilité se manifeste et la population se révolte. Les commerçants refusent de vendre et les parents retirent leurs enfants de l'école quand les cinq enfants Weiss s'y rendent. Le conseil municipal prend alors un arrêté d'expulsion puis, s'apercevant de son erreur, le fait reprendre par le garde-champêtre, escorté de villageois armés de fourches. Le conseil décide alors de racheter la maison au prix fort, chose impossible parce que la délibération ne serait sans doute pas approuvée par la Préfecture, étant données

\footnotetext{
${ }^{21}$ L'Alsace, 27 janvier 1968.

22 Rapporté par Maurice Colinon, Des inconnus parmi nous, les Gitans - S.P.E.S., 1968, p. 93.
}

les raisons de l'achat. Il est alors décidé de passer par un organisme pour procéder au rachat de la maison, quelques jours plus tard, plus de trois fois le prix de l'acquisition. Devant l'hostilité des voisins, la famille accepte la somme ${ }^{23}$. A Kaltenhouse (BasRhin) une autre affaire a, en 1970, de nombreux échos dans la presse ${ }^{24}$.

En novembre 1980 un cas similaire se produit, encore dans le Bas-Rhin, à Mertzwiller. Les familles tsiganes refusent d'envoyer plus longtemps leurs enfants à l'école tant que la municipalité refusera l'autorisation d'installer un bâtiment à usage sanitaire sur leur terrain, où les ordures ne sont même pas ramassées. Les rats prolifèrent et un tour de garde doit être organisé la nuit, quand il fait froid, pour éviter leur invasion. L'unique robinet d'eau gèle. Les Tsiganes réagissent : nous ne pouvons même plus laver nos enfants, et après on nous dit qu'ils puent. Mais l'adjoint au maire chargé des affaires sociales refuse le permis de construire des sanitaires à ces Tsiganes dont la croissance rapide risque de déformer le visage de Mertzwiller dans un proche avenir ${ }^{25}$.

Dans le Bas-Rhin encore, à Hoerdt, commune de 3200 habitants près de Strasbourg, en 1974 la famille Weiss, installée depuis une quinzaine d'années à l'écart du pays, a quitté son habitation précipitamment: l'un des fils s'est rendu

${ }^{23}$ Le Progrès-Soir, 17 octobre 1961 et autres journaux.

${ }^{24}$ Le Figaro, 29 octobre, Les Dernières Nouvelles d'Alsace, 29 octobre, Le Monde, 31 octobre, Le Figaro, 2 novembre, Les Dernières Nouvelles d'Alsace, 5 novembre, Le Figaro, 9 novembre, Le Canard Enchaîné, 11 novembre, Lutte Ouvrière, 10 novembre, Pilote, 19 novembre, Hara-Kiri, 19 novembre, etc., et des émissions télévisées.

25 Dépêche de l'AFP, 22 novembre 1980, Philippe Bodio. En août 1981, le conseil municipal démissionne pour s'opposer au maintien des classeswagons fournies pour le relogement des Tsiganes par une association locale qui prend leur défense. En juin 1981, le maire de Labégude, près de Privas, démissionne à cause des problèmes de stationnement des nomades. En juin 1980, le conseil municipal du Vivier-sur-Mer (Ille-et-Vilaine), démissionne parce que le sous-préfet refuse de faire évacuer des nomades. 
coupable d'un vol de voiture et des coups de feu ont été tirés dans la nuit contre les abris des Weiss et des menaces proférées par des hommes ayant envahi le campement. Le maire en profite, accompagné du gardechampêtre et soutenu par la population, pour brûler la baraque et une caravane avec les vêtements, matelas, etc. et faire nettoyer l'emplacement au bulldozer, après avoir tué chiens et chats. Il déclare avoir rempli son devoir de maire responsable de l'ordre et de la salubrité publique $e^{26}$. Aucune poursuite n'est intentée, aucune sanction prononcée: le maire est soutenu par la population et de nombreux maires du département. On se rappelle peut-être qu'en 1605 une décision de Basse-Alsace demande à chaque État d'organiser chaque mois des battues contre les Tsiganes. Les Weiss en fuite vont alors loger sous la tente dans une commune voisine, Drusenheim. La Préfecture, qui veut les aider, leur offre une meilleure toile de tente! Mais le maire de Drusenheim expulse la famille avec les enfants en bas âge, par six degrés au-dessous de zéro. Par un courrier du 4 mars 1974, le Procureur de la République aurait demandé au Garde des Sceaux de classer l'affaire sans suite afin d'éviter de mettre le pays à feu et à sang à cause des nomades $^{27}$. La famille doit continuer à errer. Revenue le 6 mars à Hoerdt pour demander d'y séjourner à nouveau, le maire répond en l'expulsant à nouveau. Les maires des communes voisines font de même.

Les «affaires» qui se produisent de temps à autre polarisent l'attention de tous sur quelques faits. Les jugements humanistes portés sur ces faits en font oublier d'autres, et les grandes phrases qui stigmatisent l'attitude de ceux qui sont les instigateurs des "affaires", occultent le discours devenu routinier des plus proches, maires et voisin, qui rejettent sans le savoir. Il semblerait que seuls ceux par qui le scandale arrive s'en rendent alors compte, prévenus par ce qu'on leur dit. Ils ont d'ailleurs du mal à l'admettre

26 Les Dépêches, Dijon, 5 février 1974, et autres journaux.

${ }^{27}$ Rapporté par Monde gitan, n² 29, 1974, p.2. et plaident toujours non-coupables, étonnés de se trouver soudainement accusés pour un acte somme toute très banal.

\section{L'alternance préfectorale}

Les autorités locales ont précédé les pouvoirs publics dans la politique de rejet: ce n'est, pour la France, qu'en 1504 que Louis XII expulse collectivement les Tsiganes d'une partie du royaume, alors que les villes ont pris l'habitude de les refouler. Mais ce sont aussi les autorités locales qui, au 20e siècle, conservent une attitude de rejet, bien après que les pouvoirs publics aient préconisé l'assimilation. Il y a donc constance de la politique de rejet de la part des autorités locales, du $15 \mathrm{e}$ au $20 \mathrm{e}$ siècle, reflet de la politique que souhaitent voir appliquer les populations qui font pression sur les édiles, à voir la façon dont elles s'arment au $15 \mathrm{e}$ siècle, et dont elles se constituent en comités de défense au 20e. Et si pendant plusieurs siècles l'union des politiques se faisait autour du rejet, des populations jusqu'au gouvernement, depuis quelques décennies les divergences se précisent. Dans ce contexte les administrations préfectorales apparaissent comme des relais traversés de courants contraires, avec d'un côté les maires et de l'autre les pouvoirs publics.

Les maires revendiquent la légalisation du rejet. Ils écrivent à la Préfecture, en soulignant que la commune ne dispose d'aucun terrain d'accueil, et qu'en conséquence une dérogation doit être accordée à l'obligation faite à la commune d'assurer le séjour pendant quarante-huit heures aux nomades de passage ${ }^{28}$. A Biarritz, le conseil municipal a voté une délibération demandant à M. Le Ministre de l'Intérieur d'inscrire Biarrity au nombre des communes interdites aux

28 Ce qui n'empêche pas, lors d'une même délibération du conseil municipal, de décider, par exemple, de la création d'un grand terrain de camping - nous en avons vu le cas plus haut, pour un camping de sept cent cinquante places, dans la commune du Pouliguen. 
errants sans fortune. Et si la Préfecture fait une enquête auprès des communes pour établir des aires de stationnement selon les directives gouvernementales, les maires répondent ou font répondre qu'ils n'ont pas de place, ou qu'ils ont déjà satisfait aux obligations qui leur sont imposées, par la désignation d'un terrain (souvent sans eau, éloigné, exigu, insalubre). Le conseil municipal de Grand-Quevilly (près de Rouen) adresse au préfet le résultat d'une délibération réclamant des mesures efficaces contre les nomades; les élus sont défavorables à l'intention exprimée par l'autorité supérieure d'implanter ces nomades sur des terrains situés sur la rive gauche de la Seine, et afin de limiter les possibilités d'invasion, il est décidé de clore tous les terrains sans usage appartenant à la ville. Le maire d'Helfrantzkirch (Haut-Rhin) annonce que si la sous-préfecture l'oblige à garder une famille manouche indésirable, le conseil municipal démissionnera collectivement.

Les préfets ont eux aussi une longue tradition de rejet, et à la fin du $19 \mathrm{e}$ siècle comme au début du 20e, les termes des arrêtés ne présentent pas d'ambiguïté. En 1960 encore, dans les Alpes-Maritimes, l'arrêté préfectoral du 16 juin considérant qu 'il est nécessaire de prescrire toutes mesures à l'effet de préserver l'esthétique et la salubrité publique dans les zones ainsi délimitées, interdit le séjour et le stationnement des nomades dans soixantedix-neuf communes du département. Mais si les notions floues d' esthétique et de salubrité publique préfigurent les raisons d'interdictions mises en avant dans la réglementation du stationnement des années soixante-dix, il n'en reste pas moins qu'en 1960 une femme nomade porte plainte et que, de tribunal en tribunal, l'affaire est portée devant le Conseil d'État qui donne raison à la femme contre le Ministre de l'Intérieur qui s'est substitué au préfet: Un préfet porte une atteinte illégale à la liberté individuelle en interdisant, de façon permanente et absolue, le stationnement et le séjour des nomades sur tout ou partie du territoire d'un département. Cet arrêté du 20 janvier 1965 du Conseil d'État fera jurisprudence. Pourtant les préfets, dorénavant avec des nuances, continuent de rejeter. La même année 1965, rappelons la circulaire du 1er juin du préfet des Hautes Pyrénées, adressée aux maires, dans laquelle il est question de ces nomades qui contribuent à transformer nos avenantes contrées en bidonvilles disgracieux et malpropres, et le préfet incite au rejet :

Je vous invite à fixer par voie d'arrêté - si ce
n'est déjà fait - un emplacement déterminé
pour ces stationnements et je vous conseille
très vivement d'en limiter la durée. Je vous
signale cependant que vous devez autoriser le
stationnement nécessaire, celui qui permet,
par exemple, de faire reposer pendant la nuit
les gens et les attelages, mais il vous est
loisible d'interdire les stationnements de jour
qui ont pour but de permettre aux nomades
d'exercer les activités dont ils sont censés
vivre, ou qui, prolongés pendant plusieurs
jours, donnent aux roulottes et caravanes le
caractère principal de logement.

Le 2 août 1971, le préfet de la Région des Pays de Loire, préfet de LoireAtlantique, avançant comme argument l'ouverture de deux terrains de stationnement (La Civelière à Nantes et $\mathrm{La}$ Maison David à Bouguenais), prend un arrêté adressé aux maires, commissaires de police et au commandant du Groupement de gendarmerie pour limiter à vingt quatre beures au maximum la durée des stationnements, pour toutes les catégories de populations non sédentaires, sur le territoire des trente-six communes adhérentes au Syndicat Intercommunal pour l'bébergement des populations nomades. En y mettant les formes, et quelques crédits d'aménagement, un préfet peut se permettre de renouveler impunément l'arrêté déclaré illégal du préfet des Alpes-Maritimes. L'aménagement de terrains est le prix à payer pour satisfaire à la fois l'humanisme et la technocratie des pouvoirs publics (les nomades sont logés et contrôlés sur les terrains) et la méfiance des populations locales (ils sont surveillés et rassemblés dans un enclos selon la terminologie du maire de Lille pour son propre terrain). 
Mais les préfets ne font pas toujours le jeu des maires par un rejet qu'ils suscitent. La politique du gouvernement, devenue assimilationniste dans la seconde moitié du 20e siècle, est aussi la leur. Alors qu'en juin 1965 le préfet des Hautes-Pyrénées conseille aux maires de limiter la durée du stationnement, en octobre le préfet de la Mayenne veut, à la politique de répression et d'interdiction jusqu'ici pratiquée à l'égard des populations d'origine nomade [...] substituer une politique plus comprébensiver. D'autres textes, dans d'autres départements auront un sens identique. Le 22 septembre 1980, le secrétaire général de la Côte-d'Or envoie une lettre aux maires, leur rappelant les difficultés et la nécessité du séjour des populations d'origine nomade dans leur commune, et rappelle les possibilités de création d'aires de stationnement. Un questionnaire est joint à la lettre; les maires doivent répondre à des questions sur les rapports entre la commune et les nomades, sur une éventuelle aire de stationnement des gens du voyage existant actuellement dans la commune et sur les possibilités d'aménager une aire de stationnement pour les gens du voyage. La Préfecture se situe ainsi dans la droite ligne des préconisations ministérielles $^{30}$. De même lorsque les autorités ne répondent pas à la demande des

\footnotetext{
${ }^{29}$ Voir le texte et l'analyse faite dans le cadre de la politique du stationnement, "La fin du voyage », Monde Gitan, op. cit.

${ }^{30}$ Qui apparaissent encore dans la circulaire $\mathrm{n}^{\circ} 80$ 262 du 10 juillet 1980 des ministres de l'Intérieur, de l'Environnement et du Cadre de Vie, du Travail et de la Participation aux préfets et sous-préfets. Il est demandé dans un délai de six mois de rendre compte du plan d'aménagement élaboré dans le cadre de votre département et tout particulièrement des aires de stationnenent actuellement en cours de réalisation. La Préfecture de la Côte-d'Or, bien que dans la lettre aux maires il ne soit pas fait mention de la circulaire du 10 juillet, est bien dans cette ligne. De son côté le préfet des Yvelines, par des circulaires en août et novembre 1980, demande aux maires, tout en leur fournissant des précisions (choix des emplacements, possibilités de financement), si l'implantation d'une aire de stationnement est envisageable dans la commune. Sur les 118 (parmi les 251 maires sollicités) qui font connaitre leur avis, 112 répondent par la négative et 6 par une acceptation de principe (deux élus de la majorité de l'époque, deux socialistes et deux communistes).
}

maires de faire expulser les nomades : lors d'un rassemblement religieux tsigane au début du mois de juin 1980 au Viviers-surMer (Ille-et-Vilaine) le maire fait appel aux C.R.S. pour repousser les nomades, mais le préfet n'accède pas à sa demande; les commerçants de la localité ferment alors et le conseil municipal envoie sa démission au préfet, qui la refuse, étant données les raisons de cette démission ${ }^{31}$.

\section{L'ambiguïté gouvernementale}

Les pouvoirs publics doivent régulièrement inciter les autorités locales à une politique plus «compréhensive ». En ce qui concerne les nomades, jusqu'à présent, le grand obstacle n'a jamais été l'obstacle financier (le Fonds d'Action Sociale a financé des terrains de stationnement, même au-delà de ses pourcentages habituels). Il est plus facile de trouver des crédits que de convaincre les municipalités de la nécessité de créer un terrain et que d'en choisir l'implantation ${ }^{32}$. Pour inciter, de nombreux textes ont été élaborés $^{33}$. Ils se succèdent, et reviennent tous sur la nécessité du stationnement pour les nomades, pour des raisons diversement évoquées: sédentarisation et intégration des nomades, apprentissage de la sédentarisation au moyen notamment d'une action socioéducative ${ }^{34}$. Par ailleurs, la jurisprudence fait que les maires ne peuvent interdire de manière générale et absolue le stationnement des nomades,

\footnotetext{
${ }^{31}$ Cf. Le Monde, 17 juin 1980; Libération, 16 juin 1980, etc.

32 Nicole Lafay, chargée de mission au Ministère de Travail, de l'Emploi et de la Population, Bulletin de liaison des techniciens sociaux, C.N.I.N., n 4, 1974, p. 4.

33 Déjà, en 1957, alors qu'il était peu question d'assimilation, la loi $\mathrm{n}^{\circ}$ 57-908 du 7 août tendant à favoriser la construction de logements et les équipements collectifs inscrit clairement dans son article 26 les aires de stationnement au nombre des équipements indispensables. Cette loi est complétée par le décret du 17 avril 1962, et ensuite une série de circulaires, les 8 mars 1966, 13 avril 1967, 4 août 1967, 20 août 1968, 5 février 1969, 16 mai 1978 du Ministre de l'Intérieur. 34 Ces textes ont été présentés plus en détail en rapport avec la politique du stationnement. Cf. mes autres textes publiés.
} 
mais doivent leur permettre de se reposer quarantebuit heures au minimum ${ }^{35}$.

$\mathrm{Si}$ le discours gouvernemental est fortement incitateur lorsqu'il préconise des mesures d' «accueil » des populations d'origine nomade à insérer dans les populations qui les entourent, ce discours est étayé par tout un art de la nuance qui le fait se perdre dans ses propres arcanes. Dans les rapports avec les autorités locales des ambiguïtés apparaissent souvent, et des contradictions entre les mots et les actes lorsque des décisions doivent être prises.

Dans une question écrite du 26 avril 1973, Pierre Bas, député, attire l'attention du Ministre de l'Intérieur sur l'existence illégale - dans nombre de communes, d'une interdiction de stationnement pour les caravanes des "gens du voyage», et il demande si la législation en vigueur, notamment le code de l'administration communale, donne aux préfets les pouvoirs nécessaires pour mettre fin à une telle situation, notamment en annulant ces arrêtés. Le Ministre répond que

L'article 82 du code de l'administration communale donne aux préfets la possibilité d'annuler les arrêtés municipaux. Cette disposition est traditionnellement interprétée comme impliquant que faute d'avoir annulé dans le délai du recours pour excès de pouvoir, le préfet perd la possibilité d'exercer ce droit. Cette interprétation a paru conforme tant à la nécessité de ne pas remettre en cause les situations juridiques acquises, qu'au souci de respecter les libertés reconnues aux collectivités locales. Il est certes possible de considérer que, passé le délai du recours pour excès de pouvoirs, le préfet peut toujours abroger un arrêté municipal pour illégalité ou pour inopportunité. Mais une telle solution, sur laquelle les juridictions administratives ne se sont pas prononcées, ne manquerait pas de susciter des protestations de la part des

\footnotetext{
35 Il est question de repos. Comment se fera le travail? Réponse du Ministre de l'Intérieur à une question écrite, Journal Officiel, débats parlementaires du 19 mai 1979.
}

magistrats municipaux. Aussi, les instructions du Ministre de l'Intérieur ont-elle recommandé aux préfets d'intervenir auprès des maires afin qu'ils rapportent eux-mêmes les mesures d'interdiction de stationnement qui ne seraient pas justifiées par des nécessités ou des impossibilités locales. ${ }^{36}$

La nuance est telle qu'elle dénature la légalité qui permet au préfet d'annuler un arrêté d'un maire :

68B - A l'égard des arrêtés réglementaires et
notamment des arrêtés de police, le préfet (ou
le sous-préfet) est doté d'un pouvoir
d'annulation qu'il peut exercer sans être tenu
par un délai et soit d'office, soit à la demande
de particuliers intéressés (Loi 1884, art. 95).
Il peut prononcer l'annulation pour
inopportunité ou illégalité (Conseil d'État,
16 décembre 1910: commune de Saint-
Marc, D.P. 1912.3 .141$. Conclusions
Pichat). ${ }^{37}$

$\mathrm{Au}$ nom de l'interprétation traditionnellement admise, au nom de la nécessité de ne pas remettre en cause les situations juridiquement acquises et du souci de respecter les libertés reconnues aux collectivités locales, le Ministre appuie la non-intervention des préfets et attend le bon-vouloir des maires. Ainsi une réglementation de police municipale illégale mais traditionnelle peut se maintenir et se reproduire.

Le même type d'ambiguïté dans la démarche gouvernementale apparait dans la réglementation du stationnement. L'analyse des textes qui le régissent montre l'extrême difficulté qu'un nomade éprouve dès qu'il s'agit pour lui de s'arrêter: au nom de la protection des sites, ou de la salubrité et tranquillité publiques, il est invité à

\footnotetext{
36 Journal Officiel, débats parlementaires, Assemblée nationale, du 18 juin 1973.

37 Maxime Letourneur, Droit public et administratif, Dalloz, 1959. L'auteur fut vice-président de la section contentieux du Conseil d'État. Dans des avis récents, le Conseil d'État a confirmé nettement le droit d'annulation à toute époque des arrêtés municipaux de police.
} 
poursuivre son chemin. Et stationner plus de trois mois sur un terrain de son appartenance l'oblige à déposer un dossier plus complexe qu'une demande de permis de construire. Le décret du 4 septembre 1980 relatif au camping, au stationnement des caravanes et à l'implantation d'habitations légères de loisirs s'ajoute aux dispositions précédentes et rend kafkaien l'arrêt des nomades $^{38}$. Aux mesures qui réglementent spécialement le stationnement des "gens du voyage » viennent s'ajouter, paradoxalement, pour eux qui ne sont généralement pas acceptés dans les terrains de camping, la réglementation dorénavant complexe qui touche les caravaniers. Il y a donc une fois de plus à l'égard des nomades et Tsiganes superposition de deux réglementations : celle qui leur est spécifique et une autre, plus large, qui les englobe aussi sans reconnaitre leur spécificité, situation qui les met d'avance et inéluctablement, pour de nombreux cas, dans une situation illégale. Entre autres conséquences, il résulte du nouveau décret que des interdictions anciennement prévues pour la création de terrains aménagés (ce qui représente une infrastructure et une permanence qui peuvent justifier la prudence et les difficultés administratives) sont étendues au stationnement, même isolé et bref, de toutes les caravanes. Le stationnement devient interdit dans les sites classés ou inscrits, dans les zones définies par la loi de 1913 sur les monuments historiques classés ou inscrits ou proposés pour le classement, dans les zones de protection établies en application de la loi du 2 mai 1930 sur la protection des monuments naturels et des sites, dans les bois, forêts, parcs classés par un plan d'urbanisme ou un plan d'occupation des sols comme espaces boisés à conserver.

\footnotetext{
38 Décret n 80-694 du 4 septembre 1980, Journal Officiel, 7 septembre 1980, pp. 2121-2122. Ce décret modifie surtout le code de l'urbanisme, mais aussi les décrets des 7 février 1959 et 9 février 1968 relatifs au camping et les décrets des 25 mai 1968 et 18 novembre 1975 relatifs aux villages de vacances. Une longue circulaire du 13 mars 1981 s'ajoute au décret du 4 septembre 1980 et en renforce les tendances.
}

$\mathrm{Au}$ nom de l'écologie la réglementation interdit les écologistes pratiquants qui souhaitent utiliser la nature et les sites qu'elle propose. La réglementation protège les sites en les mettant sous une vitrine dont la transparence est illusoire. Il devient interdit de s'y arrêter. Au lieu de pénaliser des abus on interdit l'activité qui est susceptible d'y mener: c'est encore là de la prévention généralisée. Ce n'est plus le délit qui est sanctionné mais la situation dans laquelle l'individu pourrait être amené à le commettre. D'ailleurs, disposition aggravante, les infractions, qui renvoient dorénavant au code de l'urbanisme, sont des délits qui ne sont plus du ressort du tribunal de simple police et qui débouchent non plus sur une amende mais sur une condamnation pénale.

Enfin, et ici réapparaît l'ambiguité du discours des pouvoirs publics, la mise en application de la réglementation repose sur les maires et donc sur leur arbitraire qui a tendance à s'exercer au détriment des nomades de passage, alors que l'accueil des touristes en caravane est favorisé. Ce décret pris pour tous les caravaniers touche particulièrement les nomades, par l'utilisation discriminative qui peut en être faite. Les armes fournies par les pouvoirs publics aux autorités locales apparaissent ainsi comme de plus en plus diversifiées, et en contradiction avec le discours d'assimilation tenu par ailleurs.

En Belgique, comme en France, comme en Grande-Bretagne et ailleurs, les directives gouvernementales restent sans effet, et les textes parus au Moniteur Belge ne sont pas plus efficaces que ceux du Journal Officiel $^{39}$.

\footnotetext{
${ }^{39}$ Elisa et Léon Tambour, Le statut juridique des Tsiganes en Belgique, 1977, multigr. Cf. aussi le document du Conseil de l'Europe CPL/Cult (14) la partie 1Belgique: Rôle et responsabilité des collectivités locales et régionales face aux problèmes culturels et sociaux des populations d'origine nomade, 31 oct. 1979. Pascal Chevalier et Françoise Herbay, A travers la situation des itinérants, statut juridique des Tsiganes en Belgique, U.O.L.,
} 


\section{Les aires de réclusion}

La seconde moitié du 20e siècle voit s'affronter des courants divers dans le traitement des nomades et des Tsiganes. Si de la part des pouvoirs publics l'assimilation est souhaitée, avec la mise en place d'une réglementation de plus en plus complexe enveloppée d'un discours humaniste et développée pour des raisons technocratiques, le rejet de la part des autorités locales et des populations dont elles sont les élues reste brut, brutal, artisanal, sans nuance. Ces proies faciles pour la maréchaussée que sont les nomades n'ont pas besoin d'être fichées et jusqu'au seuil des années quatre-vingt le contrôle par un ordinateur central de ces populations marginales ne rassure pas assez celui qui, même de loin, voit s'installer des caravanes dans la commune ${ }^{40}$. Une loi comme celle de 1969 ne suffit pas à changer les mentalités qui en restent à celle de $1912^{41}$. L'attitude à l'égard du Tsigane n'est pas une question de loi mais d'état d'esprit alimenté par les images et représentations que chacun s'en fait.

Qu'importe les directives gouvernementales. Le rejet demeure. Il est expérience quotidienne. Dans chaque région le Tsigane est toujours un homme en sursis, voleur et fauteur de troubles en puissance, donc appréhendé en instance, éternel récidiviste par le fait même de n'avoir pas obtempéré aux textes qui depuis des siècles

Séminaire de droit international privé, 1974-1975, notamment pp. 54 et suiv.

40 Suite à la loi de 1969 a été créé un fichier national géré par la Gendarmerie nationale à Rosny-sous-Bois. Système PROSAM, mémoire FILMOREX avec micro fichiers. Au 15 avril 1976, 60858 «sans domicile ni résidence fixe » adultes sont répertoriés. Chiffre donné par M. Bertrand Cadi et le Colonel Prouteau (chef du Service Technique de Recherches Judiciaires de la Gendarmerie Nationale) dans Contribution à l'étude d'un groupe marginal dans la société francaise, IXe Congrès International de Défense Sociale, p. 10 (document non daté).

$41 \mathrm{Au}$ sujet de ces lois , J.-P. Liégeois, «Tsiganes, nomades et pouvoirs publics en France au 20e siècle », Pluriel, n¹9, 1979. l'ont banni ou promis aux galères ou à la potence. Au cours des siècles passés les autorités locales ne tenaient que rarement compte des lettres de protection du roi dont quelques groupes tsiganes étaient porteurs. $\mathrm{La}$ peur demeure au 20e siècle, et les attitudes qui en découlent.

Certains maires empilent mot à mot les griefs qui légalement leur permettent de repousser les nomades; ils pensent que leur addition donnera du poids à l'arrêté qu'ils prennent : le 16 septembre 1980, le maire de Longeault (Côte-d'Or), dans son arrêté, considère

que le campement et le stationnement sur la
voie publique d'individus n'ayant pas de
domicile fixe présentent de sérieux
inconvénients et souvent même des dangers au
point de vue de l'bygiène publique et de la
sécurité des biens et des personnes; qu'ils
gênent d'ailleurs la circulation publique et
sont une cause permanente de désordre.

L'assemblage des termes, dont aucun n'a de sens précis, induit un portrait horrifiant des maux dûs à ceux n'ayant pas de domicile fixe il convient alors de s'en protéger par une réglementation.

L'image des Tsiganes telle qu'elle s'est reproduite au cours de l'histoire de leurs contacts avec ceux qui les entourent a de quoi susciter une peur justificatrice et inspiratrice du rejet. Il est question jusqu'au 20 e siècle de "ravages et désordres", de «pilleries et saccages ». L'amalgame avec les vagabonds et gens sans aveu, avec les déserteurs armés des troupes royales et les bandits de grands chemins fait que le terme de nomade, de Gitan ou de bohémien qui demeure au $20^{\mathrm{e}}$ siècle est à lui seul porteur des exactions attribuées à ceux qu'ils ont côtoyés $^{42}$. Le stéréotype qui s'est fixé est un mélange de crainte imprécise et de superstitions à base d'histoires

42 Nous verrons ailleurs quelle est la réalité de la criminalité tsigane qui n'a rien de commun avec l'image qu'on s'en fait. 
d'empoisonnement de puits, de développement de la peste, d'incendie de récoltes, de découverte de trésors, de sorcellerie ou de dons de guérison, projection des fantasmes de l'époque où il s'est constitué, le tout présentant une sédimentation dont seules les couches les plus récentes semblent actives lors du rejet et sont utilisées pour le justifier. Les autres, enfouies, ne sont plus de mise à l'époque où la rationalité est censée dominer la réflexion. Le citadin va chercher dans le mythe les raisons de sa négation du nomade, et le rejet du paysan reste ancré sur les petits vols pas toujours réels dont la nature (poule ou pomme) importe plus que la valeur monétaire $^{43}$.

Au nom de quoi se font les expulsions? Ce n'est pas très clair dans l'esprit de celui qui rejette. Habitude qui devient légale parce que traditionnelle, comme l'a précisé le Ministre de l'Intérieur?

Pourquoi ces Messieurs les représentants de la loi viennent-ils si souvent le matin, à la première beure, nous molester brutalement, tapant sur nos caravanes? Va-t- il falloir aller jusqu'à demander [ ... ] au Ministre de la Santé Publique ce qu'il pense des conséquences que peuvent avoir ces actions barbares sur le psychisme de nos enfants et, qui plus est, de nos bébés? Nos bébés, brutalement réveillés en plein sommeil, en pleurs et remplis d'épouvante, sont profondément traumatisés, témoins de la panique générale provoquée par la violence et la brutalité de ces Messieurs. Ces Messieurs payés par nos impôts pour assurer l'ordre public. C'est un comble! Faut-il parler de

\footnotetext{
${ }^{43}$ Nous reviendrons ailleurs sur le contenu de ces représentations et images des Tsiganes et nomades, et sur leur fonction déjà esquissée à la fin de notre article concernant les pouvoirs publics, Esprit, mai 1980. Des recherches sont en cours : M. Dégrange, J.-P. Liégeois, J.Cl. Michon, Nomades et sédentaires, images réciproques, partie d'un rapport pour la Commision européenne, non publié. Elles seront poursuivies. Voir aussi, M. Dégrange, «L' apprentissage d'un stéréotype le Tzigane », Pluriel, $\mathrm{n}^{\circ}$ 23,1980 .
}

leurs façons d'inquisiteurs, de leur morgue, de leur mépris, de leur incomprébension? Et ces regards qui n'ont plus rien d'bumain quand ils nous lancent leur formule babituelle: "Allea! Debout sale racaille! » Et voilà! Papiers en mains, après vérification, leurs carnets remplis, ils nous dressent contravention $[\ldots]$ et nous ordonnent de partir sur le champ. Ont-ils fait leur travail? En tout cas, ils nous ont empêché de faire le nôtre; ils nous ont empếché de présenter et de vendre nos marchandises sur le marché. Ils ont mis une entrave à l'exercice de notre profession. Nous aimerions savoir, là encore, en vertu de quelle loi on peut empêcher un citoyen de gagner sa vie. Voudrait-on nous acculer à la misère? Pourtant, je le répète, nous sommes en règle. Alors, faut-il se poser cette question: en France, est-ce un délit d'être Gitan? Non, bien sûr ... Alors, que nous reproche-t-on? Qui osera nous le dire ouvertement? Mais ces Messieurs les exécutants le savent-ils eux-mêmes ? ${ }^{44}$

Si les élus locaux sont soucieux de l'opinion de leurs administrés, il est pourtant de courts moments pendant lesquels ils «invitent» les nomades à stationner sur le territoire de leur commune: lors des journées de recensement. Ainsi Lagardelle, dans le Lot, commune d'une centaine d'habitants, augmente sa population de plus de $20 \%$ en faisant savoir aux Tsiganes nomades qu'une pièce de vin blanc est à leur disposition le jour du recensement des populations itinérantes. Dans une commune du Centre, une "prime » de quarante à cent francs est donnée aux nomades pour le recensement ${ }^{45}$. Afin de compter les Gitans dans sa population locale, le maire de Beaumont-surDême (Sarthe), qui n'est jamais à court d'idées, avait organisé une fête en leur honneur le 1 er mars. Ceux-ci vinrent au nombre d'une cinquantaine installer leurs roulottes à l'entrée du bourg. Dans la matinée, ils assistèrent, admiratifs, au plongeon $d u$ maire de la commune dans les eaux froides de la Dême, puis ils défilèrent en chantant des cantiques

${ }^{44}$ J. Doerr, « Où vas-tu, Gitan ? », Monde Gitan, n 42, 1977, pp. 14-15.

${ }^{45}$ Rapporté par Monde Gitan, n 34, 1975, p. 19. 
dans les rues du bourg [...]. Tous furent ensuite conviés à un apéritif d'bonneur et à un banquet offert gracieusement par la commune. Dans la soirée, et pour remercier leurs hôtes, les Gitans offrirent une fête haute en couleur, dont la beauté n'avait d'égale que le pittoresque du feu de camp ${ }^{46}$. L'accueil des nomades supportés pendant une journée est politiquement fonctionnel (augmentation du nombre des habitants de la commune) alors que le rejet de tous les autres jours est électoralement nécessaire (pression des administrés).

Le dernier quart du 20e siècle s'ouvre malgré tout, pour les autorités locales, sur une nouvelle politique, avec un glissement, sous la pression des pouvoirs centraux mais avec beaucoup de retard sur eux, d'une politique d'exclusion à une politique de réclusion. Si l'exclusion se produit toujours, l'évolution montre qu'à côté du rejet coexiste la réclusion: se débarrasser, devant les pouvoirs centraux qui réglementent et devant les populations qui s'inquiètent, du « problème » des nomades et Tsiganes en les encadrant dans un terrain préparé à cet effet. Il a fallu attendre six siècles de présence en France des Tsiganes pour s'apercevoir que le rejet systématique n'a pas de sens: quand la nation ou la commune rejette, la voisine fait de même et le parcours est infini. Puis des terrains «d'accueil » ont été proposés, cachés à plusieurs kilomètres d'une route carrossable, en plein champ (comme à Lens), ou derrière le cimetière ou encore sur la décharge des ordures. Terrains sans équipement, sans $\mathrm{eau}^{47}$. Les nomades sont imaginés sales. Envoyés sur un terrain insalubre l'image que se font ceux qui les perçoivent ainsi installés ne s'améliore pas. Ils apparaissent dans les journaux locaux décrits et photographiés dans la boue et les immondices.

\footnotetext{
46 "Hier, jour 'J' du recensement - Les Gitans fêtés à Beaumont-sur-Dême ", Le Maine Libre, Le Mans, 2 mars 1968

47 Il ressort d'une enquête nationale rapportée par Monde Gitan, $\mathrm{n}^{\circ} 9,1969$, p. 26, que 42\% des lieux de stationnement désignés n'ont pas de point d'eau.
}

Le raffinement de la réglementation nationale se poursuivant, l'insistance des pouvoirs publics ne se démentant pas, le courant humaniste fin de $20 \mathrm{e}$ siècle prenant de l'ampleur, les autorités locales ont changé de comportement. Les Tsiganes ont été considérés comme des sous-prolétaires à rapprocher d'autres et dans quelques villes il a été question d'H.L.M. D'ailleurs la loi du 14 décembre 1964 qui précise les modalités d'expulsion des habitants de bidonvilles indique que le refus des intéressés du relogement qui leur est offert permet l'expulsion sans indemnité, par arrêté préfectoral. Et jamais une caravane n'a été proposée pour reloger un nomade que les circonstances avaient amené dans un bidonville. Et on a pu voir, à Tarbes, par exemple, les Tsiganes aller la journée dans les H.L.M. et rentrer le soir dormir dans le bidonville. Puis les raisons financières concernant le coût de l'aménagement de terrains pour les nomades n'ont pas pu être avancées par les autorités locales pour excuser et expliquer le rejet des nomades, puisque le gouvernement, par des subventions, a donné les moyens de la politique préconisée. Des «aires de stationnement » pour les nomades ont été aménagées.

Le gain obtenu par l'ouverture d'un terrain par une commune n'est pas négligeable, même si ce n'est qu'en ce début des années quatre-vingt que les autorités locales semblent s'en rendre compte. Il est d'ordre à la fois économique et psychologique. En Grande-Bretagne, il a souvent été remarqué que les évictions des nomades (en attelant de force leurs caravanes à des véhicules tous-terrains) et le creusement de tranchées autour des terrains libres pour empêcher les nomades de s'y introduire coûtaient aussi cher que l'aménagement léger d'une aire de stationnement. En France aussi, grillage, tranchées et pancartes métalliques atteignent des prix élevés dans un budget communal, et de plus ne sont pas subventionnables comme l'aménagement d'un terrain, sans compter les activités incessantes de la police 
pour chasser tous ceux qui stationnent. Cet aspect économique est loin d'être sans importance, même si pendant des siècles chacun payait le prix fort pour éloigner les nomades.

Le gain psychologique concerne plusieurs aspects. L'aménagement de terrains de stationnement et les raisons qui y président telles qu'elles sont données et soulignées dans le discours se situent dans le courant humaniste qui s'est développé depuis 1950. Les édiles jubilent, et leur presse s'en fait l'écho flatteur: Quimper va accueillir humainement les gens du voyage ${ }^{48}$ même s'il ne s'agit que d'une visite sur l'emplacement du futur terrain. A Lille le maire a pris une décision énergique et le bulletin municipal parle de décision méritoire en 1974, pour un terrain qui en 1981 n'existe pas encore. On parle dans tous les cas d'expérience pilote. Sans souligner que depuis déjà longtemps c'est tout simplement se mettre en règle, pour une commune, que de prévoir un terrain pour les nomades de passage. On est ainsi humaniste à bon compte, et deux autres avantages, de taille, en découlent pour les autorités locales et les populations: l'ouverture d'un terrain permet d'interdire le stationnement ailleurs, même si le terrain est trop petit, et les conditions d'ouverture, auxquelles sont associées plusieurs dispositions, gardiennage et activités socioéducatives, facilitent considérablement le contrôle policier et social.

Ce n'est jamais spontanément que les autorités locales cherchent un terrain et en proposent l'aménagement pour les nomades. C'est sous la pression du gouvernement ou pour en finir avec les réclamations des riverains face aux stationnements «sauvages », ou pour dégager des terrains publics ou privés sur lesquels les nomades avaient pris $l^{\prime}$ habitude de s'installer ${ }^{49}$. Dès

\footnotetext{
48 Ouest-France, 4 novembre 1977.

49 Voir par exemple la statistique des « raisons de l'aménagement d'un terrain » données par les autorités locales d'Angleterre, dans B. Adams, J. Okely, D. Morgan, D. Smith, Gypsies and Government Policy in
}

qu'un terrain est ouvert, même nonaménagé, le maire interdit. Par une longue circulaire du 20 août 1968 ayant pour objet les terrains de stationnement pour personnes vivant en caravanes, le Ministre de l'Intérieur, le Ministre des Affaires Sociales et le Ministre de l'Equipement et du Logement entérinent une telle attitude des maires, pour mieux les inciter à la création de terrains dans le but de sédentariser les nomades :

Les communes trouveront à la création de
terrains de passage des avantages certains.
Etant dans l'obligation de tolérer le
stationnement des roulottes et caravanes, elles
pourront du fait de la création d'aires de
stationnement, veiller à ce que celui-ci
s'effectue dans des conditions satisfaisantes
tant pour l'ordre que pour l'hygiène publics.
Car, dès lors que le stationnement aura été
autorisé à un emplacement officiellement
désigné, il sera possible de l'interdire sur tout
autre terrain communal.

Le rassemblement facilite le contrôle, policier et social. A Dijon, bien avant que le terrain soit ouvert, il était question de gardiennage dans la presse : Le rôle du gardien serait alors de veiller à ce que les installations demeurent en parfait état mais aussi aurait-il pour mission de maintenir l'ordre ${ }^{50}$. Des terrains sont de véritables pièges permettant à la police de nous surveiller et nous arrêter quand elle veut ${ }^{51}$. Et le règlement explicite de certains ne permet pas d'équivoque. Le maire de Melun (Seine-etMarne) après que la ville ait préparé en 1977 un terrain de stationnement, prend un arrêté

England, Heinemann, 1975, p. 317. Un nombre très infime de réponses introduit des raisons d'aide aux Tsiganes, ou la scolarisation des enfants. Il serait dans le même ordre d'idée utile de voir comment le nomadisme est pris en compte dans les études faites pour les Plans d' Occupation des Sols et ce qui résulte des recherches commanditées par les Sociétés d'Equipement régionales ou autres organismes semipublics.

50 «Un problème irritant, le stationnement des nomades à Dijon », Les Dépêches, 3 janvier 1968.

51 Communiqué envoyé à la presse par «Un groupe de Manouches du Val d'Oise »en janvier 1977, et publié par Libération sous le titre « Partout nous nous heurtons au racisme». 
pour réglementer le stationnement sur le nouveau terrain; car il ne suffit pas qu'un outil de qualité soit créé, encore faut-il qu'il fonctionne dans des conditions valables. Cet arrêté constitue d'ailleurs - et chacun en apprendra la portée et la grande utilité une charte très complète et très stricte $d u$ stationnement et du campement des roulottes et caravanes à Melun ${ }^{52}$. Considérant, indique le maire, que le campement et le stationnement de toutes les personnes sans domicile fixe, présentent de sérieux inconvénients et souvent même des dangers du point de vue de l'bygiène publique et de la sécurité des biens et des personnes, qu'ils gênent d'ailleurs la circulation publique et sont une cause permanente de désordre, l'arrêté interdit le stationnement ailleurs que sur le terrain prévu, de toutes les personnes sans domicile fixe, voyageant soit isolément, soit en bandes. Sur le terrain, la durée du campement ne pourra dépasser quarante-buit heures. L'heure d'ouverture est fixée à 8 heures, celle de fermeture à 19 heures. Dès leur arrivée les usagers doivent passer au burean d'accueil, y prendre connaissance du règlement, payer une taxe, déposer une caution et décliner leur identité en présentant les pièces justificatives. Le gardien représente en permanence le maire de la ville et peut constater les contraventions au présent arrêté. Une tenue correcte est exigée et aucune parole, chanson on attitude ne doit être susceptible de choquer les voisins, en particulier les enfants ${ }^{53}$. L'usage de la radio, de la télévision ou de tout autre instrument sonore n'est toléré que dans la mesure où il ne gêne pas les voisins, et le silence est de rigueur entre 22 heures et 7 heures; mais dès 21 heures et avant 8 heures toutes activités bruyantes, les chants, les appels doivent être évités ainsi que le bruit des transistors.

Les plans des terrains, surtout s'ils sont réalisés par les services d'architecture de la ville, montrent le rôle attribué aux «aires de stationnement»: une entrée devant la maison du gardien et un alignement de cases

\footnotetext{
${ }^{52}$ La République de Seine-et-Marne, 7 mars 1977.

53 A noter que les seuls voisins, dans le bois entre le terrain et la route nationale, sont les prostituées et les automobilistes qui les fréquentent. Les dames sont venues demander aux nomades de ne pas laisser vagabonder les enfants dans le bois, afin de ne pas être gênées dans l'exercice de leur tâche.
}

d'environ cinq mètres sur dix. Entassement fonctionnel des caravanes destinées à être vues dans la normalité de leur alignement. Quelquefois le centre social est situé juste au centre, émergence d'où la vision du tout est possible $^{54}$.

Organiser un terrain, c'est projeter sur le sol
une certaine idée; c'est inscrire une conception
dynamique des rapports humains, sous forme
de pierres et de bois. Le Voyageur à qui nous
nous adressions ne nous disait que son désir
de liberté et nous l'invitions à décrire les
limites de sa servitude, nous l'obligions à
imaginer une liberté réduite à quinze mille
mètres carréss.

On est loin de l'organisation spontanée de l'espace par les usagers. La conception et la fonction des terrains de stationnement conçus par les municipalités apparaissent comme des mesures qui, tout en répondant aux préoccupations gouvernementales - humanisme et contrôle au cours d'un processus d'assimilation satisfont les populations et collectivités locales inquiètes - enfermement dans un secteur délimité ${ }^{56}$. En cela le vide juridique apparu par la complexité de la réglementation du stationnement est comblé dans la pratique. La règle est simple: interdiction de stationner en dehors des zones délimitées.

Renversement des perspectives: le stationnement, d'interdit dans certaines zones à préciser, devient interdit partout sauf dans quelques aires contrôlées. Les disciplines

\footnotetext{
54 On peut se rappeler les descriptions faites du panoptisme de la prison par M. Foucault, dans Surveiller et punir, Gallimard, 1975, telles que nous y avons fait allusion dans «Le discours de l'ordre. Pouvoirs publics et minorités culturelles », Esprit, mai 1980, pp. 17-50.

55 Bernard Provot, «Le terrain de stationnement des Molines à Angoulème. Chronique de quatre saisons ", Etudes Tsiganes, $\mathrm{n}^{\circ} 2$, 1974, p. 37.

56 Par exemple le titre de La République de Seine-etMarne du 7 mars 1977, au sujet du terrain de Melun, est: Tant attendu ... le terrain municipal pour les nomades entre en service: il évitera le stationnement anarchique des roulottes.
} 
établissent une "infra-pénalité" ; elles quadrillent un espace que les lois laissent vide; elles qualifient et répriment un ensemble de conduites que leur relative indifférence faisait échapper aux grands systèmes de châtiment $^{57}$. Mais disciplines et loi se rejoignent. L'effet de surveillance de la politique d'enfermement des autorités locales est renforcé par la nécessité d'action socio-éducative et de gardiennage décrite et préconisée dans la réglementation en tant qu'elle est l'élément indispensable et indissociable de l'aménagement d'un terrain, au point que les subventions les plus importantes en dépendent:

\section{Le Fonds d'Action Sociale ne peut intervenir dans le financement de la construction et de l'équipement d'une aire de stationnement que si les deux conditions ci-après sont effectivement remplies : - mise en place d'un système de gardiennage [...] - existence sur l'aire de stationnement d'une infrastructure valable rendant possible une action socio- éducative [...] Ces deux conditions excluent donc expressément le concours financier $d u$ Fonds d'Action Sociale pour la construction d'aires légères de type terrain de passage, d'aires non-gardées ou sans possibilité d'action socio- éducative ${ }^{58}$.}

Le socio-éducatif humanise l'interdiction et normalise le marginal alors déviant banalisé. Les terrains de stationnement se transforment en aires de réclusion. La liberté de mouvement des "personnes d'origine nomade» $y$ est surveillée, et les terrains deviennent des réserves exigües : avec les Tsiganes, chacun aura son Indien chez soi, qu'il pourra visiter, revenant en cela à la première attitude des Mâconnais en 1419 qui se succèdent la nuit autour du camp tsigane pour satisfaire leur curiosité, comme le feront les Parisiens en 1427, avant de les expulser. Des échoppes seront peut-être admises par les

\footnotetext{
${ }^{57}$ M. Foucault, Surveiller et punir, op. cit ., p. 180.

58 Circulaire du 10 juillet 1980 relative aux modalités de financement d'aires de stationnement pour les gens du voyage. Voir aussi la circulaire du 20 février 1968.
}

municipalités complaisantes et commerçantes (taxes). D'autres terrains seront des purgatoires où séjourner avant d'entrer dans le paradis de la maison en dur et des H.L.M. Ils sont quelquefois dénommés terrains de transition, cités de transit des nomades récalcitrants qui ne s'accommodent pas des murs. Près d'Albi l'aire de stationnement des nomades s'appelle le «terrain du Séquestre ».

Le refus du nomade est la chose du monde la mieux partagée par les populations sédentaires et les autorités locales. Il reste lié à l'image que les populations se font des nomades et des Bohémiens, à peu près identique depuis plusieurs siècles. Ce qui varie dans le discours qui sous-tend la politique, ce sont les éléments sciemment mis en avant pour justifier le traitement appliqué aux Bohémiens et nomades. Si la peur du brigandage s'est atténuée, on la trouve dans l'image de façon masquée et quasi-mythifiée avec le développement de l'urbanisation. Il reste que le Tsigane qui arrive dans une commune est toujours à la fois étrange et étranger, «sans aveu ». Il est sans domicile ni résidence fixe, on ne connaît pas son origine et on l'accuse de masquer son identité et de changer d'état civil au gré des circonstances, de perturber la tranquillité et la salubrité publiques. La marginalité du nomade inquiète d'autant plus qu'elle n'est pas clairement, comme d'autres, marginalité d'importation récente, ou séquelle de colonisation. $\mathrm{Ni}$ immigré pour sa main-d'œuvre, ni coloré, sans pays d'attache, le Bohémien n'est pas compris par les repères habituels. S'il inquiète les pouvoirs publics en tant que dissident susceptible par son existence même de troubler l'ordre d'un consensus précaire, pour les populations et les autorités locales il réactive des fantasmes liés à l'équilibre psychologique des communautés.

Le nomade est insaisissable et même une place périphérique lui est refusée: l'ouverture du terrain de stationnement évitera surtout que les nomades [...] ne séjournent à la lisière, voire an cxur même des quartiers 
périphériques ${ }^{59}$. La marginalité périphérique est trop vague, et seul le nomade banalisé rangé sur un terrain agréé peut être l'objet de l'aide des Bureaux de bienfaisance, devenus Bureaux d'Aide Sociale des communes. L'idée n'est ni nouvelle ni spécifique: à certaines époques seuls les pauvres porteurs d'un badge de reconnaissance étaient secourus par la communauté, et les vagabonds étrangers étaient expulsés ou incarcérés $^{60}$. A défaut de pouvoir repérer suffisamment le nomade, les communes cherchent à l'éloigner sans nuance. Le Journal $d^{\prime}$ administration des communes rurales ${ }^{61}$ publie deux modèles d'arrêtés, dont l'un interdit totalement le stationnement des nomades (donc il est illégal), accompagné d'un commentaire: Le seul moyen vraiment efficace d'empêcher les exactions des nomades est assurément d'interdire leur séjour, au risque de commettre une illégalité et de voir l'arrêté annulé. Mais qui osera en faire la demande? Le consensus local pousse au rejet. Interdiction de stationner, et même interdiction de circuler; ainsi dans le Cher: Route départementale $n^{\circ} 82$ - Circulation interdite aux nomades. Il arrive aussi que les Tsiganes servent d'enjeu politique, comme à Lille et Lambersart où les municipalités de gauche et de droite se combattent et s'accusent depuis des années par Tsiganes interposés au sujet du stationnement ${ }^{62}$, ou à Toulouse, quand en 1971 un tract électoral est distribué au sujet du terrain de Ginestous où sont installés les Tsiganes :

\section{Louis Bazerque veut transférer le camp de Ginestous dans votre quartier (chemin de Gabardie). \\ Le terrain est acheté. Si Bazerque est élu, les travaux commenceront prochainement.}

Pierre Baudis s'est toujours opposé formellement à ce projet si néfaste pour tous.

\footnotetext{
59 A Melun, La République de Seine-et-Marne, 7 mars 1977.

${ }^{60}$ Cf. G. Taylor, «The problem of poverty 1660 1834 », Seminar Studies in History, London: Longmans, 1969, p. 146, cité par N. Castan, «La justice expéditive », Les Annales, n 2, 1976, p. 352. ${ }^{61}$ Janvier 1966.

${ }^{62}$ Voir J.-P. Liégeois, «Expulser les nomades, le cas de Lille » Esprit, mars 1981.
}

La décision dépendra de votre vote les 14 et 21 mars.

Aidez. Pierre Baudis à sauvegarder la tranquillité de votre quartier!

Et quand il y a un "problème » qui risque de se transformer en affaire et que la publicité rend indéniable, ou lorsqu'une étude «sérieuse » du problème est réalisée, les divers partenaires se renvoient la balle : Il $n^{\prime}$ y a pas de solution policière, car la solution est d'ordre politique et incombe à Messieurs les Maires $^{63}$. Le problème des Gitans, c'est une affaire d'État, déclare le maire de Meaux ${ }^{64}$ alors que les municipalités sont réticentes pour adopter les préconisations des circulaires ministérielles en matière de stationnement. De son côté la préfecture dira que c'est aux municipalités de faire procéder si nécessaire aux expulsions ${ }^{65}$. Pour les maires, les nomades sont affaire d'État, et pour l'État, c'est l'affaire des maires.

A l'examen de l'attitude des autorités locales vis-à-vis des Tsiganes et nomades, on s'aperçoit qu'elles servent de tampon entre les pouvoirs publics dont la politique a tourné au milieu du 20ème siècle pour souhaiter l'assimilation, et des populations locales restées rejetantes. Les préfets, après avoir encore joué le jeu des autorités locales, depuis 1970 environ, un grand nombre de circulaires les ayant rappelés à l'ordre dans la décennie précédente, s'alignent sur la position gouvernementale et sollicitent les maires pour l'aménagement des terrains de stationnement. Et peu à peu, les autorités locales, de tampon deviennent relais dans les dernières années du 20e siècle, les politiques s'homogénéisent et les communes

\footnotetext{
63 Lettre du 9 février 1972 de M. Chessel, Commissaire de Police de Villeurbanne Nord à Monsieur le Contrôleur Général, Chef du District de l'Agglomération Lyonnaise, citée dans l'étude réalisée par Acide 1901, Implantation d'aires de stationnement pour les Gens du Voyage, Région de Lyon, 1977, p. 56.

${ }^{64}$ Le Parisien Libéré, édition de Seine-et-Marne, 15 avril 1968.

65 Préfecture de l'Essonne, «Pas de halte à Grigny pour les gens du voyage », Le Monde, 13 juin 1978, p. 41.
} 
comprennent fort bien que les réglementations du stationnement, de la circulation et des professions des Tsiganes et nomades, convergent pour le maintien d'un ordre, d'une tranquillité et d'une salubrité publique dont elles seront tout autant bénéficiaires que les pouvoirs centraux. Les dissensions entre maires et préfets, et le passage par le tribunal administratif, ne sont qu'escarmouches entre gens de même compagnie, qui finissent par s'accorder, et que le temps fera s'accorder. À partir de 1980 , le rejet des uns et l'assimilation des autres n'existent que dans une opposition de forme, et non dans une opposition de fond. La solution finale qui satisfait la nécessité du contrôle et l'humanisme du discours est la fin du voyage : chaque famille nomade aura trouvé place dans la case d'un terrain agréé ; la famille, dont les métiers reposaient sur la mobilité, deviendra assistée socialement tandis qu'elle sera assimilée culturellement ${ }^{66}$. Le rejet du nomade cessera faute de nomades à rejeter; mais il apparaîtra sous d'autres formes, liées à la ségrégation sociale.

Jean-Pierre Liégeois Centre de Recherches Tsiganes Université Paris V

\section{Au $21^{\text {ème }}$ siècle : une régression}

Entre chacun des paragraphes du texte précédent, rédigé il y a trente ans, il serait possible d'inscrire un nouveau paragraphe, fondé sur l'actualité de 2010. Il serait facile de le faire : à l'époque, internet n'existait pas, et les ordinateurs personnels n'étaient pas encore accessibles; aujourd'hui internet nous apporte quotidiennement une profusion d'informations qui pourraient aisément illustrer et confirmer les analyses présentées. Il serait d'ailleurs important d'apporter ce complément, car cela permettrait de mettre en lumière et de mesurer tant les permanences et les similitudes que les différences, d'analyser ainsi les discours, leur fonction en relation avec les attitudes adoptées et les comportements qui en découlent, les fonctionnements et dysfonctionnements institutionnels, les hiatus ou les accommodements entre les différents niveaux du pouvoir, en bref cette microphysique du pouvoir comme la nommait Michel Foucault, pour laquelle les Tsiganes, Roms ou «Gens du voyage » sont de bons analyseurs ${ }^{67}$.

Par exemple dans le texte ci-dessus, j'écrivais :

Il arrive aussi que les Tsiganes servent
d'enjeu politique, comme à Lille et
Lambersart où les municipalités de gauche et
de droite se combattent et s'accusent depuis
des années par Tsiganes interposés au sujet
du stationnement ${ }^{68}$.

67 J'en ai analysé les raisons dans plusieurs textes, notamment depuis 1977 dans «Une autopsie du travail social de prévention ", in Idéologie et pratique du travail social de prévention (J.-P. Liégeois, dir.) Editions Privat, Toulouse, 1977, pp. 13 à 22, «Travailleurs sociaux et minorités culturelles », in ibid. pp. 23 à 136 ou encore «Le discours de l'ordre, pouvoirs publics et minorités culturelles » in Esprit, Paris, mai 1980, pp. 17 à 44.

68 Voir J.-P. Liégeois, "Expulser les nomades, le cas de Lille » Esprit, mars 1981.
66 La possibilité de nomadisme est pour les populations tsiganes un élément de cohésion et de dynamisme culturels. Cf. J.-P. Liégeois, «Les Tsiganes, culture et nomadisme », Cultures, UNESCO, $\mathrm{n}^{\circ}$ 2, 1977, pp. 89-103. Le titre anglais est aussi révélateur : «Gypsies : cohesion within dispersion ». 
Or que publie La Voix du Nord, en juillet 2010 ?

Au père Arthur, qui lui téléphonait cette
semaine, l'adjointe lilloise à la solidarité a
opposé un niet catégorique. Lille n'a pas
vocation à accueillir toute la misère du monde,
et en prend déjà sa part, selon la socialiste. ""
On a 1200 places d'hébergement, $30 \%$ de
l'agglomération. C'est toujours pareil, on
amène la pauvreté là où elle est déjà. Le père
Arthur peut aller demander à Bondues,
Marcq-en-Baroeul ou Lambersart. " (...)
Moralité, quand Lambersart virera à gauche
et qu'Aubry présidente ira négocier une
solution européenne à Bruxelles, les Roms
seront sauvés.

Depuis plus de trente ans la partie de ping-pong se poursuit ainsi entre des villes voisines, avec une balle qui s'appelait «Nomades », devenue "Gens du voyage », puis «Roms». Ainsi «le rejet séculaire» se poursuit, qu'il soit, comme indiqué plus haut, simple, violent ou indirect. Les aires dites «d'accueil», quand elles existent, car leur nombre est notoirement insuffisant, restent dans leur conception des aires de réclusion. Rappelons qu'elles étaient conçues en vue d'une assimilation des populations concernées, de leur apprentissage de la vie sédentaire $^{70}$. L'article analyse aussi ces ruptures brutales dans la gestion de l'espace, par exemple lors du basculement des règles d'urbanisme, quand le stationnement, d'autorisé partout sauf dans les lieux interdits, devient interdit partout sauf dans les lieux autorisés. Véritable révolution, surtout quand les autorisations sont octroyées de façon différentielle, ce qui signifie souvent discriminatoire dès lors qu'il s'agit des «Gens du voyage » : la caravane du touriste n'est pas traitée comme celle du « Gens du voyage ».

\footnotetext{
69 «Abriter des Roms dans une ex-école : la ville rejette la prière du père Arthur », Dimanche 04.07.2010, 05:08 - La Voix du Nord.

70 «Le discours de l'ordre, pouvoirs publics et minorités culturelles »op. cit.
}

La situation actuelle n'est donc pas meilleure. De plus les travailleurs sociaux, instruments de la politique d'assimilation mentionnée, qui avaient une approche humaniste et un projet pédagogique ${ }^{71}$, sont souvent remplacés aujourd'hui par des sociétés de gestion, autant dire de gardiennage, qui se partagent, à la suite des appels d'offres lancés par les communes, un marché lucratif. Leur cahier des charges et leurs objectifs ont le mérite d'être explicites : surveillance, sécurité, contrôle, protection des infrastructures, respect du règlement, perception des taxes de séjour et paiement des fluides. Le bien-être et la satisfaction de la clientèle ne sont pas souvent pris en compte. La législation et les réglementations vont aujourd'hui encore plus loin, et à la fin des années 2000 le contrôle social est recouvert par le contrôle policier.

J'écrivais dans l'article que

\section{Le dernier quart du 20e siècle s'ouvre malgré tout, pour les autorités locales, sur une nouvelle politique, avec un glissement, sous la pression des pouvoirs centraux mais avec beaucoup de retard sur eux, d'une politique d'exclusion à une politique de réclusion. Si l'exclusion se produit toujours, l'évolution montre qu'à côté $d u$ rejet coexiste la réclusion.}

L'observation s'est vérifiée, et dans le dernier quart du $20^{\text {ème }}$ siècle le mouvement qui allait de l'exclusion à une inclusion synonyme d'assimilation, ou à la « réclusion» par une relégation spatiale et la surveillance sur les terrains, a effectivement dominé. Les autorités locales s'inclinaient peu à peu devant les directives nationales menant à une intégration spatiale et sociale des dénommés "Gens du voyage ». Certes, comme je l'ai publié ailleurs, la volonté d'assimiler n'a jamais réduit le désir d'exclure, et les populations locales ont encore bloqué l'application de lois telles que la loi Besson de 1990, qui voulait favoriser un accueil, en s'inspirant de textes qui l'ont précédés,

${ }^{71}$ Voir Idéologie et pratique du travail social de prévention, op. cit. 
comme cité dans l'article, ou encore la loi Besson de 2000, dont le même objectif n'a été atteint que de façon très partielle. Il serait sans doute utile d'avoir une loi Besson 2010, puisqu'en plusieurs décennies les lois n'ont pas été appliquées.

Mais le $21^{\text {ème }}$ siècle s'ouvre sur une autre tendance. Si la fin du siècle précédent voyait une convergence des pratiques d'assimilation, le début du $21^{\text {ème }}$ siècle voit la convergence des pratiques d'exclusion. Ce ne sont plus les collectivités locales qui entrent dans la dynamique d'assimilation préconisée par les États, mais ce sont les États qui s'inscrivent dans une politique générale d'exclusion. Chacun renoue avec le rejet séculaire présenté au début de l'article. Et au niveau national les États signent des accords bilatéraux, pratiquent des reconduites à la frontière, ne respectent pas les engagements internationaux qu'ils ont ratifiés.

Il conviendrait aussi d'analyser l'impact, depuis les années 1990, puis plus encore dans les années 2000, de la mobilité de nouvelles familles roms migrantes, peu nombreuses statistiquement parlant, mais qui suscitent de fortes réactions des populations et des différents niveaux de l'administration et de la politique, avec une instrumentalisation de cette présence, hypertrophiée dans les discours et exacerbée dans les actes ${ }^{72}$. Un brouillage se développe, pour le public, les administrations, les responsables politiques. Qui est «Gens du voyage », néologisme administratif récent, abstraction globale sans singulier, ni réalité sociale? Qui est Tsigane? Qui est Rom?

Ce brouillage ne facilite pas le respect des droits des populations concernées, car il induit des amalgames, des clivages, des confusions, entre des citoyens nationaux, des citoyens communautaires, des citoyens

\footnotetext{
72 Sur ces points, et pour une contextualisation globale, on peut voir les deux ouvrages de synthèse : Roms en Europe, Editions du Conseil de l'Europe, 2007, et Roms et Tsiganes, Editions La Découverte, Collection « Repères » série « sociologie », Paris, 2009.
}

européens, des membres d'une même minorité, des réfugiés, des demandeurs d'asile, la liberté de circulation, la liberté d'établissement, le regroupement familial, l'exercice des professions, etc. Les enquêtes réalisées, sous la forme d'études d'opinion pour ce qui est des représentations sociales, ou de statistiques pour ce qui est des violences à l'égard des personnes, montrent que les Roms sont en Europe les premières victimes de la discrimination. Des indicateurs précis existent aussi par les instances ou instruments de suivi mis en place notamment par le Conseil de l'Europe: Commissaire aux Droits de l'Homme, Commission européenne contre le racisme et l'intolérance (ECRI), Cour européenne des Droits de l'Homme, Comité européen des Droits sociaux, qui assure le suivi de la Charte sociale européenne.

Pour ce qui est du thème de cet article, sur la circulation et l'accueil des nomades, rappelons que la France est considérée comme ayant violé plusieurs des articles de la Charte sociale européenne :

Comité européen des Droits sociaux
Numéro / Intitulé 51/2008 - Date
d'enregistrement 17/04/2008
Décision sur le bien-fondé - Date 19 octobre
2009
Conclusion Violation des articles 31 \1 et 2,
16, 30 et $19 \$ 4 c$ violation de l'article E
combiné avec les articles 31, 16, 30 .

Non-respect du droit effectif au logement des gens du voyage entrainant leur exclusion sociale et une discrimination raciale (nombre insuffisant d'aires d'accueil, conditions de vie ne répondant pas aux normes minimales, absence de possibilités d'accès à des logements permanents et absence de garantie de maintien dans les lieux, absence de mesure pour remédier aux déplorables conditions de vie des migrants Roms).

(i) violation de l'article 31』1 de la Charte révisée (unanimité); 
a) en raison de la création insuffisante d'aires d'accueil;

b) en raison des mauvaises conditions de vie et des dysfonctionnements des aires d'accueil ;

c) en raison de l'accès insuffisant au logement des gens du voyage sédentarisés;

(ii) violation de l'article 31』2 de la Charte révisée en raison de la procédure d'expulsion et des autres sanctions (unanimité);

(iii) violation de l'article $E$ combiné avec l'article 31 de la Charte révisée (12 voix contre 2);

(iv) violation de l'article 16 et de l'article E combiné avec l'article 16 de la Charte révisée (unanimité);

(v) violation de l'article 30 de la Charte révisée (unanimité);

(vi) violation de l'article $E$ combiné avec l'article 30 de la Charte révisée (11 voix contre 3);

(vii) violation de l'article $19 \int 4 c$ de la Charte révisée (unanimité). ${ }^{73}$

Suite à cette décision sur le bien-fondé rendue par le Comité européen des Droits sociaux dans la réclamation $n^{\circ} 51 / 2008$, où il a été conclu que la France ne respecte pas le droit au logement des "Gens du voyage », le Comité des Ministres a adopté la Résolution Res/CM/ChS(2010)5 le 30 juin 2010. Le Comité des Ministres

1. prend note de la déclaration $d u$ gouvernement défendeur indiquant que la France poursuit ses efforts dans le domaine en cause et s'engage à mettre la situation en conformité avec la Charte révisée ;

2. attend de la France qu'elle fasse rapport, lors de la présentation du prochain rapport relatif aux dispositions pertinentes de la Charte sociale européenne révisée, sur la mise en cuure des mesures annoncées, et qu'elle tienne le Comité des Ministres informé régulièrement de tout progrès réalisé.

\footnotetext{
${ }^{73}$ Chacun des points est largement développé dans le document, et aborde en détail la non application de la Loi Besson, la contradiction avec la mise en œuvre de la Loi Sécurité et Liberté, etc. Tous les documents sont en ligne sur le site du Conseil de l'Europe.
}

La France est donc sous surveillance; après avoir pris des engagements elle doit se mettre en règle. En France même, des instances comme la HALDE, après un long silence, ou encore la Commission nationale consultative des droits de l'Homme, commencent à mentionner le caractère discriminatoire de certains textes, et demandent au gouvernement de les modifier.

Je citerai, pour terminer, quelques lignes de l'un des Points de vue du Commissaire aux Droits de l'Homme du Conseil de l'Europe, concernant le thème ce ce numéro ${ }^{74}$ :

Ces derniers mois, un certain nombre de familles roms ont, dans plusieurs pays européens, été expulsées de leur logement par la force, la décision d'expulsion étant le plus souvent prise par les autorités locales. Les locataires n'étaient pas prévenus suffisamment de temps à l'avance et aucune alternative réelle ne leur était proposée. Plusieurs de ces expulsions constituent une violation manifeste des normes européennes et internationales en matière de droits humains (...)

J'ai aussi en connaissance d'expulsions pratiquées ou planifiées dans d'autres régions de la Fédération de Russie et en Bulgarie, en République tchèque, en France, en Turquie et au Royaume-Uni. Dans certains cas, les destructions des maisons et des biens se sont accompagnées de violences et de propos racistes (...)

Les modalités d'élaboration et d'application de ces décisions doivent être conformes aux normes des droits bumains et aux garanties procédurales communément acceptées.

En raison de ces normes, les expulsions forcées ne peuvent être pratiquées que dans les cas exceptionnels et d'une manière

\footnotetext{
74 «Les expulsions forcées de familles roms doivent cesser » Thomas Hammarberg, Point de vue, 04/09/06. Les documents du Commissaire sont disponibles sur le site : www.commissioner.coe.int.
} 
raisonnable. Toute personne concernée doit avoir la possibilité de s'adresser à un tribunal pour faire examiner, avant leur exécution, la légalité des expulsions prévues. Cette possibilité implique à la fois l'existence de recours juridiques et d'un accès à une assistance judiciaire. Des alternatives à l'expulsion doivent être recherchées dans le cadre d'une consultation authentique avec les personnes concernées. Par ailleurs, une indemnisation et un relogement adéquat doivent être proposés en cas d'expulsion forcée.

Les normes ci-dessus s'appliquent aussi aux autorités locales. Le fait que les décisions injustifiées soient parfois prises an nivean local n'exempte pas les gouvernements centraux des responsabilités découlant de leurs obligations internationales. Les États doivent superviser et le cas échéant réglementer l'action des autorités locales.

Les mécanismes de contrôle de la Charte sociale européenne ont déjà identifié plusieurs pays qui n'ont pas respecté leurs obligations internationales en matière de droit au logement pour les Roms. Par ailleurs, la Cour européenne des droits de l'Homme a jugé que les conditions de logement médiocres pouvaient, dans certains cas, être considérées comme des violations de linterdiction de la torture et des traitements inbumains et dégradants contenue dans la Convention européenne. Le Comité des Nations unies contre la torture a adopté une position similaire.

Les autorités nationales, régionales et locales doivent prendre des mesures dès maintenant. Dans une recommandation en date de 2005, le Comité des Ministres du Conseil de l'Europe a donné à tous les États membres des directives claires sur l'amélioration des conditions de logement pour les Roms. Plutôt que d'expulser des familles roms, il convient de respecter leur droit à un logement convenable. A cette fin, il est indispensable qu'une réelle consultation soit organisée avec les Roms eux-mêmes.
On constate donc qu'on reste aux « rejets éternels». Avec de plus une évolution paradoxale: alors que tout était fait, dans la seconde moitié du $20^{\text {ème }}$ siècle, pour sédentariser les nomades, au début du $21^{\text {ème }}$ tout est fait pour que le nomadisme se développe $^{75}$. Le nomadisme, pour les Roms, au cours des siècles, a été une adaptation à des conditions d'existence changeantes et difficiles. Aujourd'hui, plus que jamais peutêtre, il est réactif plus que proactif, et répond au rejet, à des expulsions, à des destructions de logements, à des menaces parfois mises à exécution (Kosovo par exemple), à une impossibilité d'accès à des ressources économiques, etc. Dans tous ces cas le Rom est contraint d'aller voir ailleurs si l'accueil est meilleur. On le rend ensuite responsable de la situation dont il est victime.

Jean-Pierre Liégeois Sociologue

Université Paris 5 - Sorbonne Lean-Pierre.Liégeois@paris5.sorbonne.fr

\footnotetext{
75 Pour un développement et une contextualisation voir Roms en Europe, et Roms et Tsiganes, op. cit.
} 